\title{
Pancreatic sarcomatoid carcinoma: An unusual evolution
}

\author{
Sahir Omrani, Mohamed Hajri, Wael Ferjaoui*, Rami Guizani, Ghofrane Talbi, Lasaad Gharbi, Rached Bayar and Mohamed Tahar \\ khalfallah \\ Department of General surgery, Mongi slim hospital, Tunisia
}

\begin{abstract}
Sarcomatoid carcinoma of the pancreas is a very rare pancreatic tumor that usually has a poor prognosis. However, we report here an exceptional case of long term evolution of this malignancy. The patient was a 73-year-old man who was operated of a sarcomatoid carcinoma of the pancreas 10 years ago. He received adjuvant chemotherapy with gemcitabine. Our patient was followed-up regularly. A routine check-up revealed that the patient was free of tumor recurrence and metastasis during 10 years. After this period of recovery, the patient presented a colonic obstruction due to a metastatic disease. Indeed, the early stage and the radical treatment may enhance the prognosis of sarcomatoid carcinoma. Also, this report demonstrates a rare presentation of pancreatic cancer with colonic metastatic obstruction.
\end{abstract}

\section{Introduction}

Sarcomatoid carcinoma is an extremely uncommon tumor that occurs rarely in the pancreas. It is characterized by the existence predominantly or exclusively of atypical spindle cells with evidence of epithelial differentiation. It is an aggressive malignancy with a poor prognosis. Its average of survival after diagnosis is of 6 months [1].

We report an exceptional case of sarcomatoid carcinoma of the pancreas that showed a very long-term evolution exceeding 10 years. After this time, the patient developed colonic metastases of his pancreatic cancer.

\section{Case report}

A 73-year-old man, with no past medical history, was operated for a tumor of the tail of the pancreas. The computed tomography scan showed a mass measuring $10 \mathrm{~cm}$, located in the tail of the pancreas invading the spleen and the stomach (Figure 1).

During the surgery, an en bloc resection of the tail of the pancreas, the spleen, a part of the stomach was done. His postoperative course was uneventful. The surgical specimen was a pancreatic tail with the attached spleen and a portion of stomach and colon. Histologically, the tumor showed a well-differentiated ductal adenocarcinoma with a sarcomatoid component in some other areas. This sarcomatous component was comprised of undifferentiated short cells with anhyperchromatic nuclei. Some occasional osteoclast-like giant cells were found. There is no infiltration of the colon or the stomach wall. The immunohistochemical studies showed that the osteoclast-like cells were positive for CK19 and CK7. The patient received post operative adjuvant chemotherapy with gemcitabine. A routine check-up revealed that the patient was free of tumor recurrence and metastasis. He consulted, 10 years later, with symptoms of intestinal obstruction. Physical examination and biology were normal. Computed tomography scan showed multiple round lesions in many segments of liver suggestive of hepatic metastases with wall thickening of the sigmoid colon (Figure 2). He was operated. Intraoperatively, we found many nodules of carcinosis, many hepatic metastases and a stenosing sigmoid mass. Sigmoidectomy and biopsy of peritoneal nodules were done. Pathological examination showed recurrence of sarcomatoid carcinoma.

\section{Discussion}

Sarcomatoid carcinoma of the pancreas is rare tumor which constitutes 2 to $7 \%$ of exocrine pancreatic neoplasm [2]. It is characterized by the proliferation of spindle cells with evidence of epithelial differentiation. Indeed, sarcomatous elements present both epithelial markers and ultrastructural features. This is explained by the influence of transforming growth factor beta (TGF beta). This factor is responsable of the metaplasia of carcinoma into mesenchymal

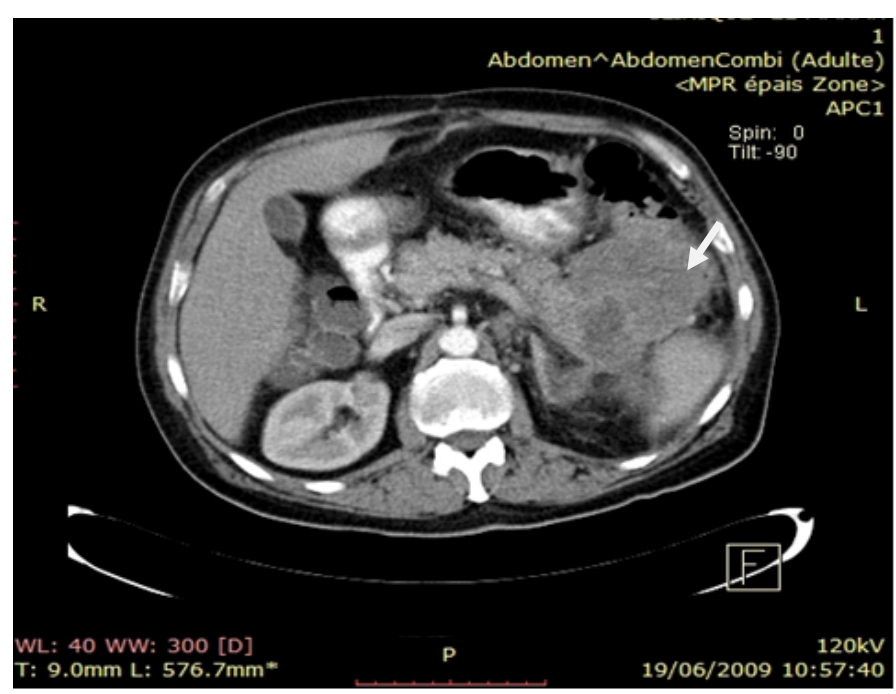

Figure 1. Arterial phase computed images sowing a low density mass in the pancreatic tail invading spleen, estimac and the splenic flexure (white arrow)

${ }^{*}$ Correspondence to: Wael Ferjaoui, Faculty of Medicine, Department of General Surgery, Mongi Slim Hospital Sidi Daoued, 1006 Tunis, Tunisia, Tel: +216 52430099; E-mail: farjaouiwael4@gmail.com

Key words: evolution, pancreas, sarcomatoid

Received: February 11, 2020; Accepted: February 25, 2020; Published: February 28,2020 

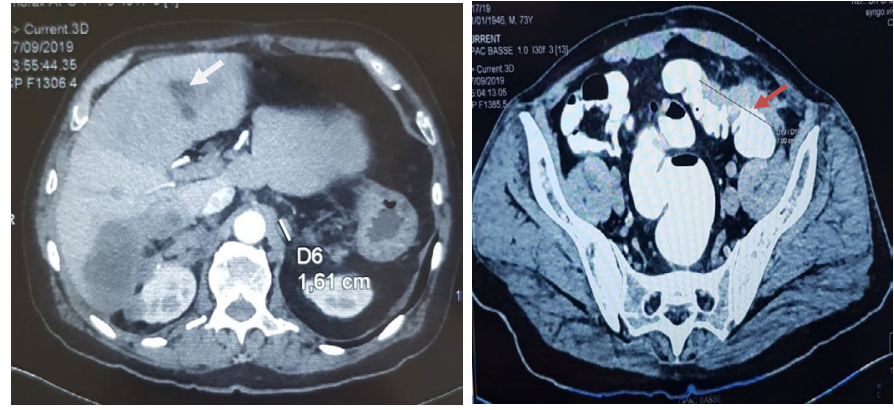

Figure 2. Arterial phase computed tomography image showing multiple hepatic metastases (white arrow) and nodules of carcinosis and wall thikening of the sigmoid (red arrow)

components [3]. In most cases, there are no histologic features of a specific line mesenchymal differentiation. However, some reported cases in the literature showed specific mesenchymal differentiation [4] (such osteosarcoma andrhabdomyosarcoma). Sarcomatoidcarcinoma is more common in males with a peak in frequency during the 7th and 8 th decades [5]. Clinical symptoms are similar to pancreatic ductal adenocarcinomas [5]. They include epigastralgia, weigh loss vomiting and even jaundice when the tumor invade the common bile duct. However, tumor markers (CEA and CA19-9) are negative in more than $80 \%$ of cases [6]. Computed tomography scan is the main imaging examination for the diagnosis. It showed a large mass (average diameter $6.5 \mathrm{~cm}$ ), heterogeneous, well circumscribed containing large areas of necrosis, located in the body or tail of the pancreas. It is associated with frequent invasion of vessels and adjacent organs and a high frequency of synchronous lymph node and liver metastases [5]. In magnetic resonance imaging, sarcomatoid carcinoma showed low signals in T2 imaging [7]. This aspect is due to the presence of a sarcomatous component which causes tumor hypoperfusion [5]. Some authors have shown the possibility of confirming the diagnosis by biopsy performed under echoendoscopy [5]. Radical resection is the principal treatment for sarcomatoid carcinoma [3]. Chemotherapy can be administrated for patients with lymph nodes metastases [3]. Sarcomatoid carcinoma has a poor prognosis. Indeed, the average life expectancy is 6 months [1]. The longest living patient, reported in the English literature, survived for 36 months [8]. In our case, the patient survived for 10 years after surgery which is very exceptional and becomes the longest living patient having sarcomatoid carcinoma of pancreas. This case shows also an uncommon presentation of colonic obstruction caused by metastasis of pancreatic cancer. Only few cases were reported [9].

\section{Conclusion}

Sarcomatoid carcinoma is a rare aggressive tumor with a poor prognosis. With an early stage and early eradication of tumor, the evolution may be exceptionally favorable with long survival.

\section{Acknowledgments}

None.

\section{Conflicts of interest}

None.

\section{References}

1. Hartwig W, Hackert T, Hinz U, Hassenpflug M, Strobel O, et al. (2009) Multivisceral Resection for Pancreatic Malignancies: Risk-Analysis and Long-Term Outcome. Ann Surg 250.

2. Paal E, Thompson LDR, Frommelt RA, Przygodzki RM, Heffess CS (2001) A clinicopathologic and immunohistochemical study of 35 anaplastic carcinomas of the pancreas with a review of the literature. Ann Diagn Pathol 5: 129-140.

3. Xie Y, Xiang Y, Zhang D, Yao X, Sheng J, et al. (2018) Sarcomatoid carcinoma of the pancreas: A case report and review of the literature. Mol Med Rep 18: 4716-4724.

4. Giordano G, Berretta R, Silini E (2016) Primary pure spindle cell carcinoma (sarcomatoid carcinoma) of the ovary: A case report with immunohistochemical study. Diagn Pathol 11: 70

5. Boudabbous M, Chtourou L, Amouri A, Grati A, Mnif L, et al. (2014) Carcinome anaplasiquesarcomatoïde du pancréas. A propos d'un cas. $J$ Afr HépatoGastroentérologie 8: 246-249.

6. Strobel O, Hartwig W, Bergmann F, Hinz U, Hackert T, et al. (2011) Anaplastic pancreatic cancer: presentation, surgical management, and outcome. Surgery 149: 200-208.

7. Lu BC, Wang C, Yu JH, Shen ZH, Yang JH (2014) A huge adenosquamous carcinoma of the pancreas with sarcomatoid change: an unusual case report. World J Gastroenterol 20: 16381-16386.

8. Ren CL, Jin P, Han CX, Xiao Q, Wang DR, et al. (2013) Unusual early-stage pancreatic sarcomatoid carcinoma. World J Gastroenterol 19: 7820.

9. Park DY, Krishnamurthi S, Chahal P, Downs-Kelly E, Morris-Stiff G (2019) Pancreatic metastases to the colon: an unusual cause of colonic obstruction. BMJ Case Rep 12: e228578.

Copyright: (C2020 Omrani S. This is an open-access article distributed under the terms of the Creative Commons Attribution License, which permits unrestricted use, distribution, and reproduction in any medium, provided the original author and source are credited. 